\title{
A Study on Autonomous Wireless Sensor Networks
}

\author{
Aiman J. Albarakati, \\ Department of Computer Engineering, \\ College of Computer and Information Science, \\ Majmaah University, Majmaah, Saudi Arabia
}

\begin{abstract}
Autonomic computing (AC) is a gifted progress to convene fundamental necessities in the devise of wireless sensor networks and its doctrine can be functional to competently administer nodes function and optimize network resources. Wireless sensor networks (WSN's) are fetching trendy in civilian and military applications e.g. monitoring, inspection, home automation, disaster recovery and several others. More or less any sensor network application entails some structure of autonomic functionality and self-configuration. IBM's inventiveness in regards to Autonomic computing loads of protocols and architectures for network self-organization and administration/management have been projected and being executed or incorporated. This research article illustrates the idea of Autonomic Computing.
\end{abstract}

\section{Keywords}

WSN's, Autonomic Computing, Sensor Networks

\section{INTRODUCTION}

Wireless sensor networks (WSNs) comprise networks made up of devices outfitted with sensing, processing, storage, and wireless communication potential. Each network node can have numerous sensing units/modules, which are capable to carry out measurements/assessments of physical variables e.g. luminosity, temperature, humidity and so on. WSN's have significant applications in the commercial, medical, scientific and military realm. Illustrations of these applications comprise smart homes and offices, environmental monitoring, intelligent transportation systems (ITS) and surveillance. In addition it has considerable practice in biomedical area. As communal dependence on WSN's technology boosts, we can look forward to the dimension and intricacy of individual/solitary networks as well as the number of networks to rise vividly. WSN's are normally utilized in extremely vibrant and intimidating settings with no human subsistence and for that reason; they ought to be lenient to the breakdown and hammering of connectivity of individual nodes. Sensor nodes ought to be smart to recuperate from malfunctions with least human participation. Networks ought to hold up practice of self-directed configuration of connectivity, addressing and routing constitution. Latest studies on Autonomic Networking can dish up as source for drawing of Autonomic WSN's. In this article we bring in Autonomic computing and WSN's perception. We also confer how the primary characteristics of Autonomic computing (AC) act in accordance with the indispensable design/devise necessities for WSN's.

\section{AUTONOMIC COMPUTING (AC):}

\subsection{Know-how}

Autonomic computing passes on to the self-governing distinctiveness of distributed computing resources, acclimatized to erratic alterations whilst hiding builtin/inherent intricacy to users and operators. Computing devices striking boost and also improved computing faculty and involvedness pooled with popularity of internet resulted in exceptional expansion in assorted network applications and networks as well. With such mounting system intricacy, network administration concerns and communication protocols are getting a height ahead of human aptitude to administer and shelter so the constancy of contemporary systems, infrastructure and data is at a progressively bigger jeopardy to undergo outages and common poor orders. Prospective network algorithms call for better robust, adaptive and scalable with flattering distributed and self-governing architectures. Computerization/automation, self-preservation and self-administration of spacious networks may perhaps unravel the dilemma till some degree. As the perception of self-administration entrenched, the most undeviating motivation one can reflect of was the autonomic role of the human central nervous system, where autonomic controls make use of motor neurons to propel circuitous messages to organs at a subliminal intensity. These messages standardize breathing, temperature, and heart beat rate devoid of full deliberation. Inspection and scrutiny of these multifaceted adaptive systems became a key source of encouragement to propose algorithms for self-managed or self-administered, self-configuring, self-organized and self-sheltered systems. Taking motivations and notions from autonomic nervous system of the human body IBM fashioned a base for autonomic systems by taking steps towards Autonomic Computing (AC) for providing ease to humans from the load of administrating computer systems which is increasing very much till the scope of un-manageability [1].

\subsection{Autonomic System (A.S)}

A system which performs tasks, manage and configure itself on predefined rules and gained knowledge without any human interaction in light of pre-defined procedures and set of rules over the time. Following are the four functional fields for selfmanagement of Autonomic System illustrated by IBM [3]:

- Self-Configuration: Automatic or routinely configuration of its components/units.

- Self-Healing: Automatic discovery or exploration and correction/reformation of irregularities/problems.

- Self-Optimization: Automatic monitoring, observation and command over resources to guarantee the proficient functioning.

- Self-Protection: Upbeat identification and protection from uninformed assaults.

\subsection{Architecture of IBM Autonomic Computing}

[02] Describe a theoretical information outline for selfadminister IT systems. In the information framework/outline, 
an autonomic system is an anthology of autonomic components. Each autonomic element/component comprises an Autonomic Manager (AM) and the Managed Resource (MR). The communication between the AM and the MR is accomplished all the way through the MR's managing interfaces, which renders two sorts of sensors, hooks and effectors. The sensors are utilized by the Autonomic Manager to get hold of the interior position of the Managed Resource and the effectors are utilized by the Autonomic Manager to modify the action of the Managed Resource. The Autonomic Manager facilitates self-management of the resource utilizing a "Monitoring, analysis, planning, and execution" control loop, with sustaining knowledge of the management policies, computing environment and several other allied reflections.

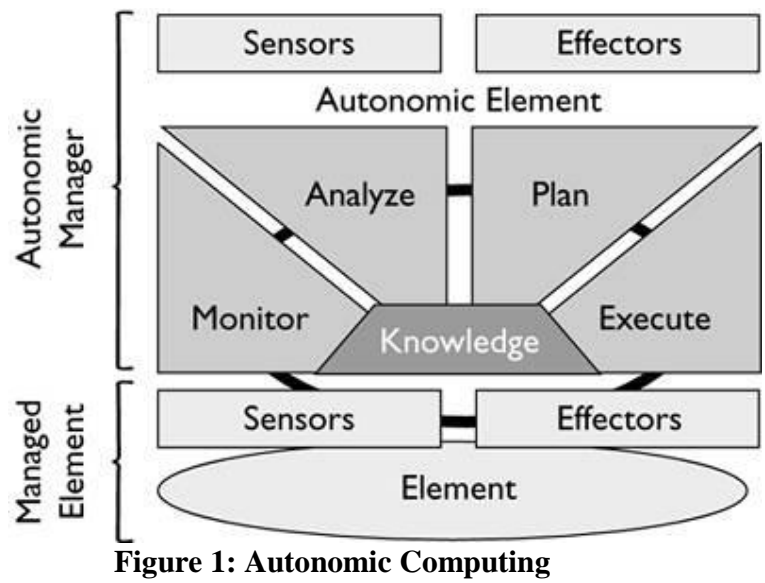

The A.C information model merely endows with the theoretical direction on scheming self-managed systems. In practice, the information model obliges to be mapped to an executable managing and organized structural design for Autonomic Networks. Exclusively, extent practices, rule engines, scheduling methodologies, dynamic resource allocation schemes, and protection and administration methods have to be developed for autonomic elements, and a scalable management platform is obligatory to harmonize the autonomic elements/components into a self-managing system.

\section{AUTONOMIC COMPUTING \& WSN's}

To shed light on the involvement that A.C is able to take in to WSN's so let's scrutinize how WSN devise necessities/requirements and maneuvers can be embark upon utilizing autonomic principles/ethics. There can be sensor nodes which are stirring and can amend their place animatedly or even abscond the network coverage vicinity. Consequently, a pre-programmed configuration for the network will not be able to work. Self-configuring nodes is capable of setting up network connections, appraise if there are any gaps/breaches in the WSN and substitute a stirred or departed/dull node within network. In view of the fact that sensors can be set up in an unattended field such as deep-sea and forest or physically inaccessible area such as inside a building wall, they are mandatory to maneuver with the bare minimum assist from base stations or individual supervisors. Even though mainstream of up to date sensor application have by now measured this in their network plan, there is at a standstill a call for WSN to have the aptitude to re-configure and pull through itself devoid of too much human interaction, particularly in unapproachable surroundings. [4][5] Sensor reading habitually has some noises; it may possibly be a counterfeit affirmative just because of breakdown of sensors. Sensors are obliged to cooperatively self-heal (e.g. identify and eradicate) false positives in their sensor readings as an alternative of pushing them to base stations. This can also lessen power utilization of sensors for the reason that data dispensation inside the sensor acquires much less power utilization than data transmission does [10]. Sensor nodes are usually uncovered to greatly harsher circumstances than typical computing equipment, and are therefore question to energy exhaustion and incidental smash up. Battery breakdown can upshot in gone astray sensor node. This escorts to a slow but sure deprivation of the network as individual nodes are vanished. Network paths shatter and gaps emerge in the coverage area. A Wireless Sensor Network has to settle in to the alteration, recuperate from fatalities and be self-sheltered. This can only be accomplished by renegotiating monitoring voltage levels, network routes inside sensor node, controlling each node by a base station or an agent and upon breakdown triggering superfluous nodes to reinstate smashed ones, or by enlightening a number of sophisticated entities which can endow with support. Utmost effectiveness has to be achieving from the on hand energy as the obtainable energy at every sensor node is restricted. Sensing, Processing and data transfer phases entail much energy as a result each node ought to be able to sense process and transfer data astutely for this reason self-optimization is an imperative feature for Wireless Sensor Networks protocols. Savings of energy can be accomplished by positioning the nodes into a squat power sleep mode, geared up to be reactivated when the call for crop up. E.g. sensors may possibly drop off their duty cycles when there is no considerable alteration in their sensor readings. This fallout in smaller amount power utilization in the sensors. Also, when adjoining sensors account ecological alteration, a sensor may possibly draw implication from the reports and raise its duty cycle to be further vigilant for an impending limited ecological amendment in the prospect. Nevertheless, trade-off exists in the computational outlay of a globally-optimal way out for example this is frequently computationally inflexible, whether by 8 -bit nodes or 64-bit base-stations. The entire fundamental WSN self-management principles act in accordance with the perception of autonomic computing. As a result an IBM autonomic computing principle is able to be applied to wireless sensor networks to acquire the preferred functionality in immensely mounting sensor network applications.

\section{MANAGEMENT ARCHITECTURES OF AUTONOMIC WIRELESS SENSOR NETWORK}

The fundamental A.C model barely endows with the theoretical guidance on scheming self-managed systems and oblige to be mapped to an implementable/executable management and control structural design for Autonomic Networks. A structural design for networking and Autonomic communication is an area of research not long and several architectures are projected and being fashioned. All these architectures endeavor to construct an architectural plan that facilitates supple, vibrant and completely autonomic configuration of across-the-board networks in which the functionalities of every constituent network node are also unruffled in an autonomic manner. Furthermore, these architectures also hold up mobile nodes and manifold 
executive domains so these can be made functional to WSN,s for pulling off preferred objectives and convene aforementioned defies. Following is the concise debate of a number of hallucinations for the blueprint of well-organized management architecture for Wireless Sensor Networks.

\subsection{Service-Oriented Architecture (S.O.A)}

[21] SOA is a progressive step towards building distributed systems that brings in application functionality/capability as services/obligation to end-user applications or to construct additional services. It decays the design/plan of outsized multifaceted application and middleware architecture into a variety of re-usable services or occupation units/modules. In Service-Oriented Architecture the service supplicant has no acquaintance of the procedural facts of the supplier's execution, e.g. the programming language, employment platform, and so on. The service supplicant usually appeals to operations by means of messages "a request message and the response" rather than through the utilization of file formats or APIs. As a result, the application developers only require distressing the operational depiction of the service which consents to software on each side of the dialogue to transform devoid of blowing the other. The implementation/execution and design/devise of Service-Oriented Architecture is typically reliant on Web Services with uniform web technologies e.g. OGSA and WSDL etc. Consequently, it is not unswervingly pertinent to all of those multifaceted technologies on those resource-restricted sensor nodes. Several initial ideas have been described in [19] utilizing the notion of service semantics from Service-Oriented Architecture. All the management function units/components In MANNA assemble at the lowest level of management architecture. These units are intended with precise implementation for individual purpose in reflection of distinctive characteristics of Wireless Sensor Networks. At the top layer a service can bring into play one or more of those management functions or tasks. Dissimilar services can carve up the similar functions, but at rest distress each individual agreed facet based on the network state and guidelines acquired from Wireless Sensor Network models. In addition, Service-Oriented Architecture is able in particular to deal with Wireless Sensor Network exclusive features such mobility, heterogeneity and adaptation, and puts forward flawless management incorporation in the wireless settings. Even though the extraordinary characteristics of Service-Oriented Architecture are splendid, but there is still a great amount of study defies desired to take in hand prior to the notions of Service-Oriented Architecture can be aptly obliged into Wireless Sensor Networks.

\subsection{Policy Based Architecture}

This management has offered its stout talent to prop up the conniving of self-adaptive decentralized management service in Wireless Sensor Networks. [20] Projected autonomic communications architecture that deal with intricacy all the way through policy-based management by integrating/incorporating a collective information model incorporated with knowledge-based reasoning methods to endow with a self-governing manner. The architecture is well thought-out utilizing four discrete architectural constructs i.e. Virtual Software, Shared Information, Infrastructure and Policy. The shared information over the network is supervised all the way through a virtual software which sustain autonomic functionality for unusual heterogeneous or assorted networks and components pooled with network infrastructure which comprise network rudiments/elements and further computing devices. All these three modules are overseen by policy module. [20]

This model is based on three imperative perceptions of autonomic computing:

(1) The sharing and reprocessing of widespread information and knowledge

(2) The application of knowledge-based reasoning and machine learning to direct the alterations in action of the system.

(3) An expandable and supple supremacy model that constitutes a closed control loop that finds out from its verdicts.

Correspondingly in [19], policies portray a set of preferred manners of management components (e.g. agent and manager) for demonstrating the real-time operations. Based on managers, polices and agents can work together with each other in an accommodating way to pull off a preferred by and large management objective such as control network density, form groups of nodes and maintain the exposure of the Wireless Sensor Network area.

\section{AUTONOMIC WSN'S ROUTING PROTOCOLS}

Following are a number of illustrious routing protocols for WSN's their pros and cons and aptness for Autonomic Wireless Sensor Network's.

\subsection{Flooding}

Flooding [24] is a mature routing method utilized in WSN's that may possibly also be utilized in Autonomic WSN's. In this technique, a node propels out the acknowledged data or the management packets to its neighbors by dissemination or flooding, except an utmost number of hops for that concern packet are attained or the destination of the packets is reached. This technique pledges the deliverance of the packet to the destination/target. Nevertheless; there are some deficits and shortcomings of flooding method:

- Implosion of Data packets

- Overlapping

- $\quad$ Resource utilization

\subsection{Gossiping}

In Gossiping [25], nodes advance arriving packets to an indiscriminately chosen neighbor node. Once a gossiping node is given the messages, it advance the data back to that neighbor or to a different one indiscriminately chosen neighbor node and as a result route from source to destination is fashioned. This practice lends a hand in energy management by randomization. Even though, gossiping can work out the implosion dilemma, it is not able to pass up the overlapping crisis. On the other hand; gossiping give out information unhurriedly, this means it puts away energy at a sluggish rate, but the outlay is long-time proliferation is desired to propel messages to all sensor nodes so it may possibly not be the preeminent fit method for Autonomic WSN's.

\subsection{SPIN}

[26] projected a pool of adaptive protocols for Wireless Sensor Networks called SPIN (Sensor Protocols for Information via Negotiation. Their objective is to stay away 
from the shortcomings of flooding protocols by utilizing data negotiation and resource-adaptive algorithms. Nodes in succession of a SPIN protocol name their data utilizing sophisticated data descriptors so-called meta-data. They make use of meta-data negotiations to eradicate the communication of superfluous data all the way through the network. Furthermore, nodes using SPIN is able to base their communication assessments equally upon application-specific acquaintance of the data and upon acquaintance of the possessions that are accessible to them. This well-organized allotment of data by sensors with restricted energy acts in accordance with the Autonomic Sensor network rations and can be extremely effectual underneath small networks. SPIN has been intended based on two fundamental thoughts:

1. To maneuver competently and to preserve energy by sending metadata.

2. Nodes inside a network have to be attentive of alterations in their personal energy resources and acclimatize to these changes to expand the operating existence of the system.

SPIN has three sorts of messages, that is to say, ADV, REQ, and DATA.

- $\quad$ ADV: When a node has data to propel, it publicizes via broadcasting this message including meta-data to all nodes in the network.

- REQ: An engrossed node propels this message when it desires to be given some data.

- DATA: Data message enclose the authentic sensor data all along with meta-data header.

This is a data-centric routing protocol where the sensor nodes propel ADV message via broadcasting for the data they have to send and hang around for REQ messages from engrossed sinks or nodes. It has the following several cons in solving the concerns coupled with traditional flooding protocols

- It is not scalable.

- If the sink is concerned in too many events, this may perhaps make the sensor nodes around it use up their energy.

- SPIN's data advertisement practice is not able to pledge the deliverance of data if the concerned nodes are far away from the source node and the nodes in between are not attracted in that data.

\subsection{Directed Diffusion}

[27] Is most efficient data propagation and aggregation protocol. An application aware and data-centric routing protocol for WSN's. It endeavors at identification all data produced by sensor nodes attribute-value pairs. Directed diffusion includes a number of rudiments; first of all, naming; where assignment descriptors sent out by the Data receiver or sink are named by allocating attribute-value twosomes. Secondly, interests and gradients; the named task description comprises an attention that restrains timestamp field and numerous gradient fields. Each leaf node and intermediate nodes accumulate the interest in their interest cache. As the interests proliferate all the way through the network, the gradients from the source reverse to the sink are initiate. Thirdly, data propagation, when the source has data for the interest, it propels out the data to the interest all along the interest's gradient path which can be selected as the unswerving hop path or undeviating time path consequent from the request packet. Fourthly, after the interest (sink) initiates receiving squat rate data events, it underpins one meticulous neighbor to depict down superior class events. This characteristic of directed diffusion is accomplished by data-driven local policies. Directed diffusion lend a hand in preserving sensors energy by choosing fine paths by processing and caching data in network in view of the fact that each node has the aptitude for performing data aggregation and caching. On the other hand; Directed diffusion has its boundaries e.g. employing data aggregation entails employment of harmonization practices which is not practicable in Wireless Sensor Networks. Moreover the overhead in data aggregation engross recording information. These shortcomings may perhaps throw in to the outlay of sensor node, for this reason outlay is the substitution for recital in Directed diffusion method, which may possibly be tolerable for a number of autonomic WSN's.

\subsection{LEACH}

[28] LEACH stands for "Low Energy Adaptive Clustering Hierarchy" is a self-governing, adaptive and clustering-based protocol that employs randomized alternation/rotation of cluster-heads to consistently give out the energy load amongst the sensor nodes within network. It is based on two fundamental postulations:

a. Base station is predetermined and positioned far away from the sensors.

b. All nodes inside the network are harmonized and energy-restricted.

The initiative at the back is to outline clusters of the sensor nodes relying on the acknowledged signal potency and make use of local cluster heads as routers to direct data to the base station. The key facets of LEACH are:

- Localized synchronization and control/management for cluster set-up and procedure.

- Randomized rotation/alteration of the cluster cluster-heads or base-stations and the subsequent clusters.

- Local constriction to trim down global communication.

Although, LEACH has revealed high-quality facets to sensor networks, but it also undergoes the subsequent downsides:

- Because of long latency it's not possible to apply it to a time constrained application.

- $\quad$ Problem of Hot-Spot (Draining of power so fast).

- Un-fixed number of clusters

- It can not be applied to large sensor networks.

\subsection{PEGASIS}

[29] PEGASIS stands for "Power-Efficient Gathering in Sensor Information Systems" is a voracious chain-based power proficient algorithm. It is based on two notions i.e. Data Fusion and Chaining. It brings into play the similar method as LEACH uses. In this method, each node is able to take twist of being a head of the chain, where the chain can be fashioned utilizing voracious algorithms that are set up by the sensor nodes. It takes for granted that sensor nodes have a 
comprehensive/global acquaintance of the network, nodes are at a stop and nodes have position information about all additional nodes. It executes data fusion apart from the end nodes in the chain. It also outperforms LEACH by eradicating the overhead of dynamic cluster formation, diminishing the summation of detachments that non leader-nodes be obliged to pass on, restraining the number of transmissions and entertains among all nodes, and utilizing barely one communication to the Base Station per round.

As PEGASIS is akin to LEACH protocol, it in addition endures from same tribulations as LEACH. Furthermore, it does not range, so can't be make functional to sensor network where global knowledge of the network is not so simple to dig up.

\subsection{GEAR}

[30] GEAR stands for Geographical and Energy Aware Routing is a recursive data distribution protocol for Wireless Sensor Networks. It employs energy conscious and geologically conversant neighbor assortment heuristics to way a packet to the targeted constituency. Within that region, it utilizes a recursive geographic informed method to propagate the packet. GEAR, similar to other sensor networks protocols, fashioned in regards to some suppositions in mind:

- Sensor nodes are immobile/static.

- A subsistence of a localization system that facilitates each node to make out its present location.

- Sensor nodes are energy-restricted accompanied with location information about all other nodes.

- Bi-directional links among nodes.

It has two phases:

(1) Advancing the packets in the direction of the targeted constituency.

(2) Advancing the packets inside the targeted constituency.

Even though GEAR trims down the energy utilization for the route set up. It is not scalable and is not able to hold up data diffusion.

Based on the compatibility assessment and investigation of the offered protocols for WSN's, we are able wrap up that some of the protocols can more or less be applied for routing in Autonomic WSN's with only some amendments depending upon the network construction and performance.

By and large, there are a number of key aspects that a wellorganized routing protocol for Autonomic WSN should have are the following [31]:

Data Aggregation

- Dynamic clustering

- Threshold for sensor nodes on data transmission and dissemination

- Randomized path selection

- Mobility

- Self-configuration
- Security

\section{ON-GOING RESEARCH/RELATED WORK}

Here is a general idea of the up to date or in progress research projects based on structural design for Autonomic Network communication and Self-administration.

\subsection{ANA (Autonomic Network \\ Architecture)}

It is proposed on the intention to endow with an architectural/structural framework that consents the adjustment of and transmission between an assortment of networks, sizing from minute degree Personal Area Networks (PAN), all the way through Mobile Ad-hoc Networks and individual rationale networks e.g. Sensor Networks to comprehensive level networks, by and large the Internet. Its framework indicates how networks interrelate. It also initiates the interior perception of "network compartments". The compartment concept consent to disintegration or atomization of networks and communication systems into minor and more straightforwardly convenient units. E.g. compartments will tolerate disintegration of today's worldwide IP network into appropriate sub-networks, which will be able to manage more separately from the entire network such as a special routing or addressing format can be put into practice within each compartment. [14] A compartment put into practices the equipped regulations and directorial guidelines for an agreed communication milieu. Compartments naturally carry out tasks like registration and deprivation/degradation, identifier management, policy enforcement and Routing and resolution.

\subsection{Bison}

A three-year project financed by the European Commission (E.U). BISON intended to deal with the intricacy explosion crisis by structuring vigorous Network Information Systems (N.I.S) that are self-governing and self-healing. BISON fashioned methods and tools for crafting full-bodied, selforganizing and flexible Network Information System as ensembles of autonomous agents by taking motivation from biological procedures and methods like ant colonies for routing in superimpose networks utilizing throng aptitude, plague for aggregation, lifecycle of Dictyostelium for load balancing and invulnerable system for seek out. BISON looked at the utilization of thoughts resultant from Complex/multifaceted adaptive/supple systems (CAS) to facilitate the structure of vigorous and self-organizing information systems for employment in extremely selfmotivated network surroundings. This project projected elucidations to vital predicaments occurring in overlay/superimpose networks and mobile ad-hoc networks by mounting algorithms for topology control in sensor networks, routing in mobile ad-hoc networks all along with data aggregation and content search algorithms for peer to peer networks. [11]

\subsection{CASCADAS}

CASCADAS stands for Component-ware for Autonomic Situation-aware Communications, and Dynamically Adaptable Services is an enduring project like ANA and Haggle. The entire ambition of CASCADAS is classifying, crafting, and appraising architectures/structures and clarifications based on a general-purpose constituent sculpt for autonomic communication services; exclusively in such background autonomic service components autonomously 
attain self-adaptation and self-organization in the direction of the stipulation of adaptive/flexible and positioned communication-intensive services. CASACDAS practice is based on four main methodical ethics:

- Circumstances awareness

- Semantic self organization

- $\quad$ Self resemblance

- Autonomic component awareness around which the prospect communication services infrastructures ought to be intended and fabricated. [16]

\subsection{Haggle}

This is an innovative autonomic networking architecture planned to facilitate communication in the occurrence of sporadic network connectivity, which takes advantage of autonomic opportunistic communications. Its node architecture seizes encouragement from human communication model. [15]

The most important components of Haggle are:

- An innovative standard for autonomic communication, based on sophisticated local forwarding and susceptible to pragmatic human mobility

- An uncomplicated and commanding architecture leaning to opportunistic message relaying, and based on solitude, validation, reliance and sophisticated data handling

- An unlock setting for the effortless propagation of services and applications.

\section{RELEVANCE \& PROSPECTS}

There are loads of applications for Wireless Sensor Networks and wide-ranging. They are utilized in money-making and manufacturing applications to keep an eye on data that would be easier said than done or costly to observe employing wired sensors. Distinctive applications of Wireless Sensor Networks comprise tracking, monitoring, and controlling. A number of explicit applications are object tracking, habitat monitoring, nuclear reactor controlling, traffic monitoring, fire detection and so on. WSN's are at present being utilized for infringement exposure by forming an outskirts just about a secure area and monitoring the succession of burglars. WSN's could be more organized in Military applications like intimidating/hostile tracking and observation, emissary monitoring. Other main existing application of WSN comprise atmosphere monitoring and applications such as flood detection, animal tracking and weather forecast and prediction and profitable applications like seismic activities monitoring and prediction. Many weather forecasting websites utilize WSN technology for reclaiming weather details in distant repressed vicinities [23]. Considerable quantity of the technology and applications are by now in continuation for monitoring activities in homes all along with invasion exposure by endowing a home with an appropriate sensorladen infrastructure. WSN's are also utilized far and wide in computerization, control and Artificial intelligence (A.I) applications like Robotics. Sensor networks are progressively more being utilized in fitness/health applications for monitoring alterations/changes in patient's health, heart rate and activities. By incessantly monitoring the striving syndrome prospects for vigorously overruling to give support to the patient may possibly be acknowledged. The Ambient Assisted existing technologies are in subsistence, which utilize Wireless Sensor Networks elements to lend a hand to the patient. An artificial retina employing Wireless Biomedical sensors has been constructed in a latest research project at Wayne State University and Kresge Eye Institute. This project meant to put up an unceasingly entrenched artificial retina with satisfactory visual functionality to consent persons devoid of vision or with partial vision to see at a tolerable level [17]. Furthermore, this biomedical sensor technology can be successfully utilized to take care of diabetes, by endowing with a more reliable, precise, and less insidious technique for monitoring glucose levels. At present, to keep an eye on blood glucose levels, a lancet is utilized to hole a finger and a drop of blood is positioned on a test strip, which then examine either electronically or manually. This invariable pricking/jabbing quite a lot of times a day over a period of years can smash up the tissue and blood vessels in that particular part. As anticipated by in [17], Wireless biomedical sensors could be entrenched in the patient on one occasion. The sensor would keep an eye on the glucose levels and put out the results to a wristwatch display. Wireless biomedical sensors may possibly play an important key job in premature finding of Cancer. As conferred in [17], cancer cells give off nitric oxide, which influences the blood flow in the part nearby a tumor. A sensor with the aptitude to perceive these variations in the blood flow are able to be positioned in suspect positions. It is probable that any aberrations could be distinguished much quicker with the sensors than devoid of no sensor.

\section{CONCLUSION}

WSN's technology puts forward noteworthy talent in abundant application fields. Agreed upon the varied character of these domains, it is indispensable that WSNs carry out in a consistent and vigorous manner. We deem, WSN has demonstrated its practice in the prospect distributed computing setting. On the other hand, there are considerable amount of scientific defies and devise concerns desires to be worked out. One of the prime defy is the scheming of wellorganized network management architecture to incessantly hold up WSNs for endowing services for an assorted sensor applications. The exclusive characteristics of WSNs construct the design, plan and implementation/execution of such management architecture unusual from the conventional networks which can be contented by notion of A.C. Yet no meticulous generic network management architecture so captivating encouragement from IBMs Autonomic Computing idea and Biological neural network system a lot of unusual research projects are presently being carried out.

\section{REFERENCES:}

[1] P. Horn, "Autonomic Computing: IBMs Perspective on the State of Information Technology", Oct. 2001.

[2] IBM and autonomic computing, "An architectural blueprint for autonomic computing," April 2003. Available from the WWW: http://www03.ibm.com/autonomic/pdfs/ACwpFinal.pdf

[3] Kephart J, and Chess D, "The Vision of Autonomic Computing" Computer Magazine, IEEE, 2003.

[4] I. Akyildiz, W. Su, Y. Sankarasubramaniam, "A survey on Sensor Networks," IEEE Communications Magazine, vol. 40, Issue: 8, pp. 102-114, August 2002. 
[5] Yu Mengjie, Mokhtar H "A Survey of Network Management Architecture in Wireless Sensor Network"

[6] C. Intanagonwiwat, R. Govindan, "Directed Diffusion for Wireless Sensor Networking," IEEE/ACM Transactions on Networking, vol. 11, pp. 2-16, Feb. 2003.

[7] O'Hare G.M.P., O'Grady M.J.“Autonomic Wireless Sensor Networks: Intelligent. Ubiquitous Sensing”

[8] D. Marsh, R. Tynan, "Autonomic Wireless Sensor Networks," Engineering Applications of Artificial Intelligence, vol 17-7, pp. 741-748, October 2004.

[9] $\mathrm{Yu}$ Cheng et al., "A generic architecture for autonomic service and network management", Computer Communications

(2006), doi:10.1016/j.comcom.2006.06.017.

[10] P. Boonma, P. Champrasert "BiSNET: A BiologicallyInspired Architecture for Wireless Sensor Networks," In Proc. of the 2nd IEEE/IARIA International Conference on Autonomic and Autonomous Systems (IEEE/IARIA ICAS), Santa Clara, CA, July 2006.

[11] BISON: Biology-Inspired techniques for SelfOrganization in dynamic Networks Project. http://www.cs.unibo.it/bison/index.shtml

[12] Shen C, Pesch D.,"A Framework for Self-Management of Hybrid Wireless Networks Using Autonomic Computing Principles" In proceedings of the 3rd Annual Communication Networks and Services Research Conference (CNSR'05)

[13] Colan, M. Service-Oriented Architecture expands the vision of Web services, Part 1. June, 2004

[14] ANA: Autonomic Network Architecture Project. http://www.ana-project.org/

[15] Haggle Project. http://www.haggleproject.org

[16] CASCADAS project. http://www.cascadas-project.org

[17] Schwiebert L., Gupta S., "Research Challenges in Wireless Networks of Biomedical Sensors" in proceedings of the 7 th annual international conference on Mobile computing and networking.

[18] Autonomic Computing Wiki. http://en.wikipedia.org/wiki/Autonomic_computing

[19] Linnyer Beatrys Ruiz, "MANNA: A Management Architecture for Wireless Sensor Networks." IEEE Communications Magazine, 2003. 41(2): p. 116-125.
[20] Davy S. et al. "Policy-Based Architecture to Enable Autonomic Communications". Available from WWW: http://techpubs.motorola.com/download/IPCOM0001414 00D/IPCOM000141400D.pdf

[21] Colan, M. Service-Oriented Architecture expands the vision of Web services, Part 1. June, 2004

[22] Gianni A., Di Caro,, "BISON: Biology-Inspired techniques for Self-Organization in dynamic Networks" [23] Wireless Sensor Networks Wiki. http://en.wikipedia.org/wiki/Wireless_sensor_network

[24] W. R. Heinzelman, J. Kulik, and H. Balakrishnan, "Adaptive Protocols for Information Dissemination in Wireless Sensor Networks," in proc. ACM MobiCom '99, Seattle, WA, 1999.

[25] S. M. Hedetniemi, S. H. Hedetniemi, , "A Survey of Gossiping and Broadcasting in Communication Networks," Networks, vol. 18, 1988.

[26] J. Kulik, W. R. Heinzelman, "Negotiation base protocols for Disseminating Information in Wireless Sensor Networks," Wireless Networks, vol. 8, pp.169-185, 2002.

[27] C. Intanagonwiwat, R. Govindan, D. Estrin, J. Heidemann, and F. Silva, "Directed Diffusion for Wireless Sensor Networking," IEEE/ACM Transactions on Networking, vol. 11, pp. 2-16, Feb. 2003.

[28] W. Heinzelman, A. Chandrakasan, , "Energy-Efficient Communication Protocol for Wireless Micro-Sensor Networks," in Proc. of the 33rd Annual Hawaii International Conf. on System Sciences, pp. 3005- 3014, 2000 .

[29] S. Lindsey, C. S. Raghavendra, "PEGASIS: PowerEfficient Gathering in Sensor Information Systems," presented at Proc. of IEEE Aerospace Conference, Montana, 2002.

[30] Y. Yu, R. Govindan, D. Estrin, "Geographical and Energy Aware Routing: A Recursive Data Dissemination Protocol for Wireless Sensor Networks," UCLA Computer Science Department UCLA-CSD TR-01-0023, May, 2001.

[31] Al-Obaisat Y, Braun R "On Wireless Sensor Networks: Architectures, Protocols, Applications, and Management" Auswireless Conference 2006. 